\title{
"Mezzanità de' caratteri e dello stile"? Die tragicommedia per musica nördlich und südlich der Alpen
}

\section{„Mezzanità de' caratteri e dello stile"? The tragicommedia per musica North and South of the Alps}

Konstantin Hirschmann / dmx14@gmx.at

Institute of Musicology, University of Vienna, AT

\begin{abstract}
At the beginning of the 18th century, a few librettists in Venice tried to avoid the new trend of transferring comical opera-scenes into intermezzi by creating tragicommedie. The two principal characters were Pietro Pariati and Girolamo Frigimelica Roberti. In their argomenti the two poets dissociated themselves from each other and conceptualized quite different views on how a tragicomic libretto had to look like. Pariati exported his view north of the Alps: He was appointed poeta cesareo in Vienna where he resumed to produce six tragicomic libretti for the imperial court during carnival which were all set to music by the compositore di camera Francesco Bartolomeo Conti. The most successful work of Pariati and Conti, Don Chisciotte in Sierra Morena, was in turn exported to Northern Germany: in 1720 to Brunswick and two years later to Hamburg, where Johann Mattheson arranged Conti's score.
\end{abstract}

\section{Keywords}

tragicommedia per musica, Pietro Pariati, Francesco Bartolomeo Conti, Girolamo Frigimelica Roberti, Johann Mattheson, Gänsemarkt-Oper. 


\section{Die tragicommedia per musica in Venedig um 1700}

$\mathrm{Zu}$ einer Zeit, da in Venedig das Komische aus der Oper in intermezzi ausgelagert wurde - also am Beginn des 18. Jahrhunderts -, umgingen einige Librettisten beziehungsweise Opernhäuser in der Lagunenstadt diese neuen Entwicklungen durch Gattungsmischungen, durch tragicommedie. Der Librettist Pietro Pariati ${ }^{1}$, der als poeta cesareo am Wiener Hof von 1716 bis 1724 sechs tragicommedie verfassen sollte und dessen Gemeinschaftswerke mit dem Wiener compositore di camera Francesco Bartolomeo Conti ${ }^{2}$ ab den $1720 \mathrm{er}-$ Jahren auch in Norddeutschland in erstaunlichem Ausmaß rezipiert wurden, hob 1707 seine erste tragicommedia, Anfitrione ${ }^{3}$, im Teatro San Cassiano aus der Taufe. Im argomento zu diesem seinem ersten Gattungsbeitrag äußert sich Pariati das einzige Mal explizit zum Thema Tragikomödie. Der Anfitrione geht auf den Amphitruo des Plautus zurück, das erste uns bekannte Stück, das sich - nota bene: augenzwinkernd - als tragicomoedia bezeichnet. Bei Plautus meint diese neu kreierte Gattung, wie Botengott Mercurius im Prolog ad spectatores kundtut, ein Stück, in dem das Personal der Komödie - diesfalls der Sklave Sosia - unter das Personal der Tragödie, nämlich Könige und Götter - im Amphitruo sind dies Jupiter und der thebanische General Amphitruo -, gemischt wird.

Als Eckpfeiler seines Anfitrione, in dem - man kennt es von Molière und Kleist - Giove (Jupiter) die Form des Feldherren Anfitrione annimmt, um mit dessen Frau Alcmena eine ausgedehnte Liebesnacht zu verbringen, gibt Pariati eine „mezzanità de' caratteri e dello stile, e la mescolanza del ridicolo con il grave "4 an, also eine - wohlgemerkt: nicht pejorativ verstandene - Mittelmäßigkeit der Charaktere und des Stils sowie eine Vermischung des Lächerlichen mit dem Ernsten. Das Lächerliche wird durch den beinah omnipräsenten, in der Tradition venezianischer tölpelhafter seicento-Dienerfiguren stehenden servo sciocco Sosia und den als Sosia verkleideten Götterboten Mercurio repräsentiert; der ernste Handlungsstrang wird zuvörderst durch die eifersüchtige Göttermutter Giunone vorangetrieben, die durch allerlei Intrigen die unwissende Nebenbuhlerin Alcmena beinah auf den Scheiterhaufen bringt. Dass die Mischung der beiden Extreme lächerlich und ernst keineswegs selbstverständlich für die venezianische tragicommedia ist, zeigt sich bei einem Seitenblick auf die Textbücher des Librettisten Griolamo Frigimelica Roberti.

Eine Wohltat für jeden Musikhistoriker ist dessen schier grenzenloser Mitteilungsdrang, denn Frigimelica Roberti ${ }^{5}$ stellt seinen Texten in der Regel ausufernde, seine

1 Den umfassendsten Band zu Leben und Werk Pariatis legte Giovanna Gronda vor, vgl. GRONDA, Giovanna. La carriera di un librettista. Pietro Pariati da Reggio di Lombardia. Con saggi di B. Dooley, H. Seifert, R. Strohm. Bologna: Il Mulino, 1990.

2 Einen brauchbaren Überblick über Vita und Schaffen Contis liefert WILLIAMS, Hermine Weigel. Francesco Bartolomeo Conti. His Life and Music. Aldershot \& Hants: Ashgate, 1999.

3 Die Musik von Francesco Gasparini ist verloren, vgl. SELFRIDGE-FIELD, Eleonora. A New Chronology of Venetian Opera and Related Genres. 1660-1760. Stanford: Stanford University Press 2007, S. 279.

4 PARIATI, Pietro. Anfitrione [...]. Venezia: Rossetti, 1707, I-Rig Rar. Libr. Ven. 425, S. 8.

5 Zu Frigimelica Roberti vgl. LEICH, Karl. Girolamo Frigimelica Robertis Libretti (1694-1708). Ein Beitrag insbesondere zur Geschichte des Opernlibrettos in Venedig. München: Katzbichler, 1972. 
Belesenheit und Aristoteles- sowohl wie Horaz-Affinität zur Schau stellende Vorworte voran. In der notizia poetica zu La fortuna per dote, seiner 1704 mit Musik von Carlo Francesco Pollarolo im Teatro San Giovanni Grisostomo aufgeführten tragicomedia, führt er aus, was er unter einem tragikomischen Textbuch versteht, und zwar „un Drama, che piglia dalla Tragedia non tutta la Favola Tragica intera; mà quel solo, che di sua natura è atto di unirsi con la Comedia. E così non prende dalla Comedia una compiuta Favola Comica; mà quel tanto, che può accoppiarsi con la Tragedia, senza guastarsi l'una con l'altra. Mà come questo? Si lascia il terribile dell'una, ed il ridicolo dell'altra, e si tempra insieme il compassionevole di quella col piacevole di questa; e ne risulta un componimento acconciamente misto, ch'è attissimo à dare quel nobile piacere, che è il basso della Comedia, non è l'affanno della Tragedia. Non move il riso dissoluto, non eccita il gusto del pianto; mà rallegra con onesto solletico di giocondo divertimento. " 6

Entscheidend ist hier die völlig entgegengesetzte Konzeption zu Pietro Pariatis Anfitrione. Während Pariati zwei Extreme, das Lächerliche - den minderbemittelten Sklaven, die durch Verkleidung hervorgerufenen Verwechslungen - und das Ernste - den Alcmena drohenden Scheiterhaufen - zu mischen trachtet, kommt es Frigimelica Roberti gerade darauf an, das Lächerliche der Komödie und das Schreckliche der Tragödie aus seiner Tragikomödie herauszuhalten, er sucht die ungefährliche Mitte, den, wie er es nennt, „ehrlichen Kitzel heiterer Unterhaltung“, der eher an die ausgewogenen Stücke des Terenz als an jene des älteren, spitzzüngigen Plautus erinnert.

Diese synthetische, um Ausgleich bemühte Konzeption Frigimelica Robertis liest sich, das sei außerdem nicht verschwiegen, wie eine Paraphrase zu Giovanni Battista Guarinis gut ein Jahrhundert zuvor entstandenem Compendio della poesia tragicomica. Der Verfasser des einflussreichen Pastor fido schreibt, der Autor einer tragicomedia „dall'vna prende le persone grandi, $\mathcal{E}$ non l'azione: la favola verisimile, ma non vera: gli affetti mossi, ma rintuzzati: il diletto, non la mestizia: il pericolo, non la morte. Dall'altro il riso non dissoluto, le piaceuolezze modeste, il nodo finto, il riuolgimento felice, E' sopra tutto l'ordine Comico “.7

Frigimelica Roberti beruft sich also implizit auf Guarini und sucht wie dieser den goldenen Mittelweg zwischen Komödie und Tragödie. Pariati stellt sich unterdes in die Tradition von Librettisten wie Giovanni Francesco Busenello, denen es, wie Florian Mehltretter in seiner Dissertation Die unmögliche Tragödie für dessen Apollo e Dafne

6 FRIGIMELICA ROBERTI, Girolamo. La fortuna per dote [...]. Venezia: Rossetti, 1704, I-Rig. Rar. Libr. Ven. 398, o.S.: „ein Drama, das der Tragödie nicht die komplette Handlung entnimmt; sondern nur das, was ihrer Natur nach fähig ist, sich mit der Komödie zu vereinigen. Genauso nimmt es [das Drama] von der Komödie nicht eine vollständige komische Handlung; sondern nur so viel, dass sie sich mit der Tragödie paaren kann, ohne dass die eine oder die andere kaputtgeht. Aber wie das? Man lässt das Schreckliche der einen und das Lächerliche der anderen und vermengt das Leidenschaftliche von jener mit dem Angenehmen von dieser; daraus ergibt sich ein füglich gemischtes Werk, das fähig ist, dieses edle Vergnügen zu geben, welches das Fundament der Komödie und nicht der Schaden der Tragödie ist. Sie ruft kein zügelloses Lachen hervor und auch nicht das Bedürfnis zu weinen; aber sie erfreut mit dem ehrlichen Kitzel heiterer Unterhaltung. " [Übersetzung K.H., wie auch sämtliche folgende Zitate.]

7 GUARINI, Giovanni Battista. Compendio della poesia tragicomica [...]. Venezia: Ciotti, 1601, S. 12: „nimmt von der einen die hohen Personen und nicht die Handlung, die wahrscheinliche, aber nicht wahre Geschichte; die bewegten, aber abgestumpften Leidenschaften; die Lust, nicht die Traurigkeit; die Gefahr, nicht den Tod. Von der anderen nehme er das nicht zügellose Lachen, die manierlichen Lieblichkeiten, den erfundenen Handlungsknoten, den glücklichen Umschwung und vor allem die komische Ordnung“. 
herausgearbeitet hat, um „die scharfe Gegenüberstellung ansonsten unvereinbarer Register“8 zu tun war. Pariati trug mithin in einer Zeit, in der die venezianische Oper sich bereits in eine ganz andere Richtung bewegte, das Grundgerüst und die Topoi des veralteten dramma per musica aus dem seicento in das neue Jahrhundert hinüber, und das wohlgemerkt, während er federführend als Autor von intermezzi an der neuen Art der Spaltung von Ernst und Komik mitwirkte.

\section{„Cosa nuova e curiosa"}

Nach seinem Engagement am Teatro San Cassiano in Venedig zog es Pariati Richtung Norden, er wurde 1714 an den Wiener Kaiserhof engagiert, wo er von 1716 bis 1724 sechs weitere tragicommedie jeweils für die Faschingszeit verfasste, die allesamt vom Hoftheorbisten Francesco Bartolomeo Conti vertont wurden und eher auf die Textbücher Nicolò Minatos zurückzublicken scheinen als gesteigertes Interesse an zeitgenössischen Reformvorhaben zu bekunden. ${ }^{9}$ In Il finto Policare, dem ersten Exemplar des tragikomischen Halbdutzends, macht Pariati ebendort weiter, wo er in Venedig aufgehört hat: Der Hirte Turbone, dessen hervorstechendstes Merkmal zweifellos seine erhebliche Intelligenzminderung ist, wird durch Zufall als vermeintlicher Thronfolger Policare zum Herrscher über Messenien auserkoren, während der wahre Policare unter dem Namen Silvero seinen Untergebenen mimt. Auch hier haben wir wie neun Jahre zuvor am Teatro San Cassiano eine Dienerfigur, die wie Sosia im Anftrione gleichsam allgegenwärtig ist und in diesem Fall - als falscher Prinz - den realiter höher gestellten Personen sogar Befehle zu geben imstande ist. Um den richtigen Policare entspinnt sich unterdes gemäß dem seicentistischen venezianischen Modell der Aufspaltung in zwei Handlungsstränge eine seria-stereotype Handlung und Liebesgeschichte mit Kämpfen, Verwundungen und Täuschungen.

In seinen weiteren tragicommedie lässt Pariati das komische Element indessen immer dominanter werden und breitet es auch sukzessive auf höhere Gesellschaftsschichten aus. 1719 folgt Don Chisciotte in Sierra Morena nach Miguel de Cervantes' Don Quixote, in welchem der durch die Lektüre von Ritterromanen verrückt gewordene Protagonist bekanntlich dem niederen Adel entstammt. Zwei Jahre später stellt Pariati in Alessandro in Sidone zwei Philosophen, den Kyniker Crate und den Hedonisten Aristippo, als zankende Witzfiguren ins Zentrum des Geschehens, und auch der Titelheld, Alexander der Große, macht nicht unbedingt die beste Figur, indem er lange Zeit am Plan festhält,

8 MEHLTRETTER, Florian. Die unmögliche Tragödie. Karnevalisierung und Gattungsmischung im venezianischen Opernlibretto des siebzehnten Jahrhunderts. Frankfurt am Main u.a.: Lang, 1994, S. 36.

$9 \mathrm{Zu}$ Pariatis und Contis tragicommedie vgl. HIRSCHMANN, Konstantin. Die Tragicommedia Pietro Pariatis und Francesco Contis und ihre Rezeption in Norddeutschland am Beispiel des Don Chisciotte in Sierra Morena. Masterarbeit Universität Wien, 2016, bzw. MICHELS, Claudia. Die Karnevalsoper am Hofe Kaiser Karls VI., 1711-1740. Denen Röm: Kayserl: vnd Königl: Majestätten zur Faßnachts=Vnterhaltung Wälsch= gesungener vorgestellet. Kunst zwischen Repräsentation und Amusement. Dissertation Universität Wien und Universität für Musik und Darstellende Kunst in Wien, 2006. 
einen der beiden zum Herrscher über Sidon zu erwählen. 1722 folgt in Archelao, re di Cappadocia, was die Ausbreitung der Komik betrifft, der Höhepunkt: Archelao, König von Kappadokien, stellt sich wahnsinnig, um - wie man heute sagen würde - einem Auslieferungsantrag aus Rom zu entgehen. Er mischt sich mit enigmatischem Geschwätz in heikle Gespräche ein, während sich der zwar nicht wahnsinnige, aber doch immerhin wahnsinnig einfältige Betrüger Sinopio den Thron erschleicht. ${ }^{10}$

Der ernste Handlungsstrang, der im Don Chisciotte und im Alessandro vermutlich in weiten Teilen von Apostolo Zeno verfasst wurde ${ }^{11}$, wird in diesen Stücken vom komischen völlig erdrückt, eine Tatsache, auf welche Pariati und/oder Zeno die LeserInnen gleich im Vorwort des Don Chisciotte hinweisen: „Dalla prima parte del medesimo è tratto non solamente quello che concerne le sue avventure di fantastica errante Cavalleria, ma quello ancora che ne costituisce le parti serie le quali in questo componimento si può dire, che ne sono come l'episodio, la dove l'altre ne sono come la principale azione. "12 Die deutsche Version des Librettos verwendet als Übersetzung für „episodio“ das Wort „Zwischen=Spiele “13. Etwas überspitzt könnte man also von ernsten intermezzi zu einer komischen Haupthandlung sprechen. Nicht zuletzt deshalb könnte Apostolo Zeno in einem Brief vom Don Chisciotte auch als einer „cosa nuova e curiosa" ${ }^{\text {"14 }}$, einer neuen und kuriosen Sache, berichtet haben.

\section{"Allerley lächerliche, lebendige Posituren"}

Diese kuriose Sache scheint auch außerhalb der Wiener Stadtmauern für Aufsehen gesorgt zu haben, denn der Don Chisciotte wurde vor allem in Norddeutschland für die damalige Zeit in erstaunlichem Ausmaß rezipiert. ${ }^{15}$ Am Hagenmarkt-Theater in Braunschweig wurde diese tragicommedia zumindest in den Jahren 1720, 1721, 1732 und 1738 aufgeführt, wie vorhandene Libretti belegen. 1720 führte man den Don Chisciotte in Braunschweig italienischsprachig als dramma per musica auf, was darauf hindeutet, dass die Bezeichnung tragicommedia im Norden auf der Opernbühne offensichtlich keine

10 In den beiden weiteren tragicommedie, Creso (1723) und Penelope (1724), schlägt Pariati über weite Strecken einen merklich weniger burlesken Ton an. Man könnte vermuten, dass das Lächerlich-Machen gleich zweier Könige in manchen Kreisen des Wiener Hofs für Unmut sorgte.

11 Pariati stellt dies unmissverständlich in einem bei CAMPANINI, Naborre. Un precursore del Metastasio. Firenze: Sansoni, 1904, S. 44f., abgedruckten Brief klar: „Vi sono alcune scene serie del collega Signor Zeno, ma la maggior parte, cioè il restante è tutto mio lavoro“. („Einige ernste Szenen sind vom Kollegen Signor Zeno, aber der größte Teil, das heißt: der Rest, ist gänzlich meine Arbeit“.)

12 PARIATI, Pietro / ZENO, Apostolo. Don Chisciotte in Sierra Morena [...]. Wien: Van Ghelen, 1719, I-Mb RACC.DRAM.2304, S. 3: „Dem ersten Teil [des Romans] wurden nicht nur die Abenteuer seiner fantastischen umherirrenden Ritterschaft entnommen, sondern auch das, was die ernsten Figuren erleben, die in diesem Stück, wie man sagen kann, die Nebenhandlung ausmachen, während die anderen die Haupthandlung bestreiten."

13 PARIATI, Pietro / ZENO, Apostolo. Der Don Quixote, In dem schwartzen Gebürg [...]. Wien: Van Ghelen, 1719, A-Wn 4609-A Mus., o.S.

14 ZENO, Apostolo. 21. Al P. Pier Caterino Zeno. a Venezia. Vienna 21. Gennajo 1719. In Lettere di Apostolo Zeno [...]. Venezia: Valvasense, 1752, S. 34.

15 Vgl. hierzu HIRSCHMANN, op. cit., S. 91ff. 
Tradition hatte. Das Libretto ${ }^{16}$, dem eine deutsche Prosaübersetzung beigefügt ist, wurde deutlich gestrafft, und vor allem die Rezitative des seria-Paars Lucinda und Fernando, der einzig wirklich ernsten Figuren, fielen dem Rotstift zum Opfer. Gleich beim ersten Auftritt der beiden zu Beginn des 2. Akts wurden ziemlich genau zwei Drittel der recitativi semplici schlankerhand gestrichen. Das heißt, die ohnehin bereits zu „Zwischen=Spielen “ degradierte seria-Handlung wurde 1720 in Braunschweig noch weiter marginalisiert. 1721 wurde der Don Chisciotte dann in einer deutschen, vom Dichter Johann Samuel Müller besorgten Übersetzung ${ }^{17}$ wiederaufgenommen. Dafür mussten die Rezitative auf jeden Fall neu komponiert werden - dass diese Aufgabe der Braunschweig-Wolfenbüttel'sche Hofkapellmeister Georg Caspar Schürmann übernahm, lässt sich allerdings in Ermangelung einer Partitur nur vermuten. ${ }^{18}$

Die in unregelmäßig gereimte, zwei- bis sechshebige Verse gegossene Version Johann Samuel Müllers lag dann auch den Aufführungen an der mit dem Hagenmarkt-Theater verschwisterten Hamburger Gänsemarkt-Oper zugrunde, wo der Don Chisciotte von 1722 bis 1737 mindestens $27 \mathrm{Mal}$ aufgeführt wurde. ${ }^{19} \mathrm{Im}$ anonymen Vorwort zum ersten dort gedruckten Textbuch ${ }^{20}$, das - dem Tonfall nach zu schließen - der Feder Johann Matthesons entstammt, wird interessanterweise vor allem der Komponist Francesco Conti mit Lob überhäuft, was im Vergleich zu anderen Hamburger Libretti der Zeit eine absolute Ausnahme darstellt. Mattheson spricht von einem „Meister=Stück von poßirlicher/ und doch dabey/ in ihrer Art/ haupt=schöner Music" Contis und attestiert dem aus Florenz gebürtigen Komponisten, dass dieser „darin so künstlich zu scherzen/ und so scherzhafft zu künsteln gewust [hat], daß sich auch ein Kenner/ bloß bey dem Anblick gewisser Sätze/ des Lachens kaum enthalten "21 könne. Auch in seinem Vollkommenen Capellmeister fällt Mattheson ein ähnliches Urteil über Conti, dessen Don Chisciotte er als Idealtypus einer „Satyra“ bezeichnet: „Seine Einfälle“, so Mattheson, „führen auf dem blossen Papier fast eben die ergetzliche Wirckung mit sich, als ob man mit Augen allerley lächerliche, lebendige Posituren vor sich sähe. " 22

Eines von zahlreichen Beispielen für derartige lächerliche Posituren ist, wie zu vermuten steht, die Arie „Sono un fulmine di guerra“ (III, 10) ${ }^{23}$, in welcher Don Chisciotte, der gerade bei einer Marionettenaufführung Mauren-Marionetten zu Kleinholz verarbeitet

16 PARIATI, Pietro / ZENO, Apostolo. Don Chisciotte in Sierra Morena [...] Don Quixotte In dem Mohren=Gebürge [...]. Wolfenbüttel: Bartsch, 1720, D-W Textb. Sammelbd. 9 (7).

17 PARIATI, Pietro / ZENO, Apostolo [/ MÜLLER, Johann Samuel]. Don Quichotte In dem Mohren=Gebirge [...]. Braunschweig: Meyer, 1721, D-W Textb. 626.

18 Einen überaus prominenten Namen findet man in der Liste der SängerInnen: Der 21-jährige Johann Adolf Hasse sang den andalusischen Adeligen Cardenio.

19 Vgl. die Auflistung bei MARX, Hans Joachim / SCHRÖDER, Dorothea. Die Hamburger Gänsemarkt-Oper. Katalog der Textbücher (1678-1748). Laaber: Laaber 1995.

20 PARIATI, Pietro / ZENO, Apostolo [/ MÜLLER, Johann Samuel]. Don Quixotte in Dem Mohren=Gebürge [...]. Hamburg: Jakhel, 1722, D-B Mus. T 5-13/25 (23).

21 Ibid., o. S.

22 MATTHESON, Johann. Der vollkommene Capellmeister. Studienausgabe im Neusatz des Textes und der Noten. Hrsg. von Friederike Ramm. 2. Auflage. Kassel u.a.: Bärenreiter 2008, S. 40.

23 CONTI, Francesco Bartolomeo. Don Chisciotte in Sierra Morena [...]. A-Wn Mus.Hs. 17207. Die Partitur liegt außerdem in einem Faksimile-Reprint vor: CONTI, Francesco Bartolomeo. Don Chisciotte in Sierra Morena. 
hat, seine Kampfkraft lobt und sich als „Kriegsblitz“ anpreist. Gleich beim Einsetzen der Singstimme stechen einem die auf- und abwärts schießenden Tiraten ins Auge, ein althergebrachtes Mittel zur Schilderung von Gefechten, das bekanntlich bereits Monteverdi in seinem Combattimento nutzte; sie alludieren einerseits auf den Krieg, dienen andererseits aber auch der Darstellung von Blitzen („fulmini“) - man denke nur an die Quattro stagioni Antonio Vivaldis. Dazu erklingen Sprünge über zwei Oktaven, hämmernde Motivwiederholungen, rasende Tonrepetitionen im Stil des concitato genere, die mit sukzessive größer werdenden Sprüngen kombiniert werden. Wolfgang Caspar Printz hätte letztere vermutlich als figurae bombilantes (zusammengesetzte bombi oder "Schwermer“) bezeichnet, Figuren, von denen er ausdrücklich festhält, dass diese in der Vokalmusik nichts verloren hätten ${ }^{24}$, man könnte mithin mutmaßen, dass Conti dem Don Chisciotte diese instrumentalen Verzierungen in den Mund legte, um ihn der Lächerlichkeit preiszugeben. Allesamt präsentieren sich die in dieser Arie verwendeten Motive jedenfalls als Codes, die den ZuhörerInnen in opere serie generell Erregung, im speziellen Fall Kampf und Krieg vermitteln sollten. Es sind hier naturgemäß nicht die Figuren an sich, die Schmunzeln hervorrufen, sondern der Kontext, in dem sie Verwendung finden: Nach einem Kampf mit Marionetten wirken sie einigermaßen lächerlich - für die ZuhörerInnen „poßierlich“. Conti verwendet geflissentlich ungeeignete musikalische Mittel, um Komik zu erzeugen, ein Vorgehen, das auch später Heinrich Christoph Koch im Artikel „Komisch“ seines Musikalischen Lexikons als probates Mittel anführt, das „Gefühl des Lächerlichen“ zu erregen. ${ }^{25}$

Dass Johann Mattheson eine besondere Vorliebe für Contis Don Chisciotte hegte, lässt sich nicht nur aus dem geradezu enkomiastischen Libretto-Vorwort schließen, sondern vielleicht auch daraus, dass er die Hamburger Partitur ${ }^{26}$ entgegen der damaligen Gewohnheit ganz allein abschrieb und einrichtete. Bemerkenswert ist außerdem, dass im Gegensatz zu anderen zu dieser Zeit an die Gänsemarkt-Oper importierten Stücken keine einzige fremde Arie/Instrumentalnummer hinzugefügt wurde, das Werk wurde in seiner Gesamtheit aufgeführt, die Arien erklangen allesamt, von Mattheson transponiert. Aufgrund der Unterschiede in der metrischen Struktur von Johann Samuel Müllers Übersetzung und der musikalischen Deklamation aus Francesco Contis Partitur mussten aber selbstverständlich die Rezitative neu komponiert werden. Was bei den Rezitativen auffällt, ist, dass deren Melodie in der Singstimme deutlich sprunghafter - und damit dramatischer - verläuft als die eher fließenden melodischen Linien in den italienischen recitativi semplici Contis. Die Bassfortschreitungen der Mattheson'schen Rezitative orientieren sich zumeist aber stark am Original.

Howard Mayer Brown (Hrsg.). Photoreprod. from Vienna, Österreichische Nationalbibliothek, MS 17.207. New York: Garland, 1982.

24 PRINTZ, Wolfgang Caspar. Phrynidis Mytilenaei [...]. Bd. 2, Dresden \& Leipzig: Riedel, 1696, S. 61.

25 KOCH, Heinrich Christoph. Komisch. In Musikalisches Lexikon [...]. Frankfurt am Main: Hermann, 1802 , Sp. 872 .

26 CONTI, Francesco Bartolomeo / MATTHESON, Johann. Don Quixote. D-B Mus.ms. 4076/1. 
Die Arien wurden in Hamburg teils auf Italienisch, teils auf Deutsch gesungen, wie das bekanntlich am Gänsemarkt seit 1703 Usus war ${ }^{27}$, teils war dies aber auch dadurch bedingt, dass der Sänger des Cardenio, der Altkastrat Valentino Urbani, wie Mattheson im Vorwort berichtet, nicht des Deutschen mächtig war. Bis auf diesen in Hamburg üblichen Polyglottismus und die durch die Besetzung mit anderen Stimmlagen notwendig gewordenen Transpositionen wurden freilich erstaunlich wenige musikalische Änderungen vorgenommen. Erstaunlich einerseits, weil es den damaligen Gepflogenheiten widersprach, und andererseits, weil die von der Musikhistoriographie oft geschmähten vokalen Leistungen der Hamburger SängerInnen offenbar immerhin mit jenen der kaiserlichen Hofkapelle in Wien mithalten konnten.

Wenn Veränderungen vorgenommen wurden, dann am ehesten in der Instrumentierung. In Don Chisciottes lamento „Ach hier häng ich“ (IV, 10) fügte Mattheson klagende Traversflöten hinzu, die sich meist im Terzabstand zu den sordinierten Violinen bewegen. An den langen französischen Einfluss, der an der Gänsemarkt-Oper herrschte, erinnert der französische Violinschlüssel, in dem die flauti traversi (!) notiert sind. Darüber hinaus fehlt im Gegensatz zur Wiener Fassung der Bass, denn die tiefste Stimme wurde von Mattheson den sich vornehmlich in chromatisch fallenden und steigenden Linien fortbewegenden gedämpften violette, also Bratschen, anvertraut; die Arie wird statt vom basso nur mehr vom bassetto di viole begleitet. Möglich ist, dass sich Mattheson hier einen musikalischen Spaß erlaubte, indem er dem während der Arie in der Luft an einem Balkon hängenden Titel-Antihelden auch noch musikalisch das Fundament unter den Füßen wegzog.

Da Matthesons Partitur über 15 Jahre in Verwendung war, finden sich in ihr dementsprechend viele Bearbeitungsspuren. Kreuz-und-quer-Kürzungen, von denen die Partitur übersät ist, gehen Hand in Hand mit den Strichen, die sich im zweiten erhaltenen Hamburger Libretto ${ }^{28}$, jenem aus dem Jahr 1732, finden. Die Kürzungen, die für die ersten Aufführungen in Braunschweig 1720 und 1721 sowie für Hamburg 1722 getätigt worden waren, halfen dem mitunter etwas geschwätzigen Libretto von Pariati und Zeno sogar ein Stück weit, jene, die 1732 für die Gänsemarkt-Oper vorgenommen wurden, verstümmelten es unterdessen. Die Informationen, mit denen sich die ZuschauerInnen konfrontiert sahen, sind äußerst knapp, und bisweilen hat man das Gefühl, nur noch ein Text-Skelett vor sich zu haben. An den von Conti komponierten Arien dieses „Repertoirestücks“ - wenn ich es so nennen darf - schien sich das Hamburger Publikum aber auch 18 Jahre nach der Wiener Uraufführung noch nicht satt gehört zu haben.

27 Vgl. ZELM, Klaus. Die Opern Reinhard Keisers. Studie zur Chronologie, Überlieferung und Stilentwicklung. München \& Salzburg: Katzbichler 1975, S. 50.

28 PARIATI, Pietro / ZENO, Apostolo [ / MÜLLER, Johann Samuel]. Don Quixote in Dem Mohren=Gebürge [...]. Hamburg: Stromer, 1732. 


\section{Bibliography}

\section{Musikalien}

CONTI, Francesco Bartolomeo. Don Chisciotte in Sierra Morena [...]. A-Wn Mus.Hs. 17207.

CONTI, Francesco Bartolomeo / MATTHESON, Johann. Don Quixote. D-B Mus.ms. 4076/1.

CONTI, Francesco Bartolomeo. Don Chisciotte in Sierra Morena. Howard Mayer Brown (Hrsg.). Photoreprod. from Vienna, Österreichische Nationalbibliothek, MS 17.207. New York: Garland, 1982.

\section{Libretti}

FRIGIMELICA ROBERTI, Girolamo. La fortuna per dote [...]. Venezia: Rossetti, 1704, I-Rig. Rar. Libr. Ven. 398.

<http://daten.digitale-sammlungen.de/0004/bsb00048753/images/index.html?fip=193.174.98.3 $0 \&$ sid $=00048753 \&$ seite $=1$ \&lang $=$ de $>$.

PARIATI, Pietro. Anfitrione [...]. Venezia: Rossetti, 1707, I-Rig Rar. Libr. Ven. 425.

<http://daten.digitale-sammlungen.de/0004/bsb00048928/images/index.html?fip=193.174.98.3 $0 \&$ sid $=00048928 \&$ seite $=1 \&$ lang $=$ de $>$.

PARIATI, Pietro / ZENO, Apostolo. Don Chisciotte in Sierra Morena [...]. Wien: Van Ghelen, 1719, I-Mb RACC.DRAM.2304.

<http://www.urfm.braidense.it/rd/02304.pdf>.

PARIATI, Pietro / ZENO, Apostolo. Der Don Quixote, In dem schwartzen Gebürg [...]. Wien: Van Ghelen, 1719, A-Wn 4609-A Mus.

PARIATI, Pietro / ZENO, Apostolo. Don Chisciotte in Sierra Morena [...] Don Quixotte In dem Mohren=Gebürge [...]. Wolfenbüttel: Bartsch, 1720, D-W Textb. Sammelbd. 9 (7).

PARIATI, Pietro / ZENO, Apostolo [/ MÜLLER, Johann Samuel]. Don Quichotte In dem Mohren=Gebirge [...]. Braunschweig: Meyer, 1721, D-W Textb. 626.

<http://diglib.hab.de/drucke/textb-626/start.htm>.

PARIATI, Pietro / ZENO, Apostolo [/ MÜLLER, Johann Samuel]. Don Quixotte in Dem Mohren=Gebürge [...]. Hamburg: Jakhel, 1722, D-B Mus. T 5-13/25 (23).

<http://digital.staatsbibliothek-berlin.de/werkansicht?PPN=PPN685790371\&PHYSID=PHYS_000 $1 \& D M D I D=>$.

PARIATI, Pietro / ZENO, Apostolo [/ MÜLLER, Johann Samuel]. Don Quixote in Dem Mohren=Gebürge [...]. Hamburg: Stromer, 1732, D-B Mus. T 7 (21).

<http://digital.staatsbibliothek-berlin.de/werkansicht?PPN=PPN688402720\&PHYSID=PHYS_000 $1 \&$ DMDID $=>$.

\section{Theoretische Schriften}

GUARINI, Giovanni Battista. Compendio della poesia tragicomica [...]. Venezia: Ciotti, 1601.

KOCH, Heinrich Christoph. Komisch. In Musikalisches Lexikon [...]. Frankfurt am Main: Hermann, 1802, Sp. 872-873. 
MATTHESON, Johann. Der vollkommene Capellmeister. Studienausgabe im Neusatz des Textes und der Noten. Hrsg. Von Friederike Ramm. 2. Auflage. Kassel u.a.: Bärenreiter 2008.

PRINTZ, Wolfgang Caspar. Phrynidis Mytilenaei [...]. Bd. 2, Dresden \& Leipzig: Riedel, 1696.

ZENO, Apostolo. 21. Al P. Pier Caterino Zeno. a Venezia. Vienna 21. Gennajo 1719. In Lettere di Apostolo Zeno [...]. Venezia: Valvasense, 1752.

\section{Sekundärliteratur}

CAMPANINI, Naborre. Un precursore del Metastasio. Firenze: Sansoni, 1904.

GRONDA, Giovanna. La carriera di un librettista. Pietro Pariati da Reggio di Lombardia. Con saggi di B. Dooley, H. Seifert, R. Strohm. Bologna: Il Mulino, 1990.

HIRSCHMANN, Konstantin. Die Tragicommedia Pietro Pariatis und Francesco Contis und ihre Rezeption in Norddeutschland am Beispiel des Don Chisciotte in Sierra Morena. Masterarbeit Universität Wien, 2016.

LEICH, Karl. Girolamo Frigimelica Robertis Libretti (1694-1708). Ein Beitrag insbesondere zur Geschichte des Opernlibrettos in Venedig. München: Katzbichler, 1972.

MARX, Hans Joachim / SCHRÖDER, Dorothea. Die Hamburger Gänsemarkt-Oper. Katalog der Textbücher (1678-1748). Laaber: Laaber 1995.

MEHLTRETTER, Florian. Die unmögliche Tragödie. Karnevalisierung und Gattungsmischung im venezianischen Opernlibretto des siebzehnten Jahrhunderts. Frankfurt am Main u.a.: Lang, 1994.

MICHELS, Claudia. Die Karnevalsoper am Hofe Kaiser Karls VI., 1711-1740. Denen Röm: Kayserl: vnd Königl: Majestätten zur Faßnachts=Vnterhaltung Wälsch= gesungener vorgestellet. Kunst zwischen Repräsentation und Amusement. Dissertation Universität Wien und Universität für Musik und Darstellende Kunst in Wien, 2006.

SELFRIDGE-FIELD, Eleonora. A New Chronology of Venetian Opera and Related Genres. 1660-1760. Stanford: Stanford University Press 2007.

WILLIAMS, Hermine Weigel. Francesco Bartolomeo Conti. His Life and Music. Aldershot \& Hants: Ashgate, 1999.

ZELM, Klaus. Die Opern Reinhard Keisers. Studie zur Chronologie, Überlieferung und Stilentwicklung. München \& Salzburg: Katzbichler 1975. 